\title{
Spécificités des poids extrêmes en réanimation
}

\section{Particularities of the Extreme Body Weights in Intensive Care Units}

\author{
A. De Jong $\cdot$ S. Jaber \\ (C) SRLF et Lavoisier SAS 2015
}

L'obésité morbide se définit par un indice de masse corporelle (IMC) $\geq 40 \mathrm{~kg} / \mathrm{m}^{2}$ et la maigreur extrême ou dénutrition par un IMC $<16,5 \mathrm{~kg} / \mathrm{m}^{2}$, selon une classification établie par l'Organisation mondiale de la santé. Les « poids extrêmes », que constituent l'obésité morbide ou la maigreur, sont associés à de nombreuses maladies pourvoyeuses d'admission en réanimation. La prise en charge de ces patients en réanimation présente des spécificités diagnostiques, thérapeutiques et pronostiques.

Il est estimé que $20 \%$ de la population mondiale est actuellement en surpoids et que près de 300 millions de personnes sont obèses. Dans la population générale, l'IMC optimal en termes de mortalité se situe entre 23 et $25 \mathrm{~kg} / \mathrm{m}^{2}$. Les décès prématurés observés chez les patients obèses sont liés au développement d'un syndrome métabolique, d'une maladie cardiovasculaire, d'une dysfonction respiratoire ou d'un cancer. Qu'en est-il chez les patients obèses admis en réanimation?

L'obésité est souvent associée à des comorbidités telles qu'un syndrome d'apnée du sommeil ou un syndrome d'obésité hypoventilation (obésité morbide associée à une hypercapnie sans autre cause retrouvée). La pression intraabdominale est élevée, favorisant la formation d'atélectasies via la diminution des volumes pulmonaires. Les résistances des voies aériennes sont augmentées et l'oxygénation diminuée, alors même que la consommation d'oxygène et le travail respiratoire augmentent. Lors de la ventilation mécanique, les patients obèses ont la particularité de pouvoir présenter des pressions de ventilation élevées pouvant parfois être le reflet, non pas d'une pression transpulmonaire élevée, mais d'une augmentation de la pression transthoracique (due à la compression de la cage thoracique par l'abdomen). Pour lutter contre ces modifications respiratoires, des mesures simples existent, basées sur une ventilation protectrice. Elle débute avant l'intubation par une préoxygéna-

\footnotetext{
A. De Jong $\cdot$ S. Jaber $(\bowtie)$

Département anesthésie et réanimation B (DAR B), hôpital St

Éloi, INSERM U1046 ; CHU de Montpellier, 80 avenue

Augustin Fliche, F-34295 Montpellier, France

e-mail : s-jaber@chu-montpellier.fr
}

tion en ventilation non invasive (VNI) en position demiassise [1], permettant une amélioration de l'oxygénation [2]. L'augmentation de l'incidence de la ventilation et de l'intubation difficiles chez le patient obèse morbide nécessite l'application d'une procédure d'intubation difficile [3], basée sur un algorithme incluant des dispositifs spécifiques d'intubation, tels que les vidéolaryngoscopes [4]. Cela a pour but de prévenir les complications de l'intubation, qui surviennent dans plus de la moitié des intubations difficiles chez les patients obèses admis en réanimation [5]. Ensuite, après l'intubation, des volumes courants bas (entre 6 et $8 \mathrm{~mL} / \mathrm{kg}$ ) calculés en fonction du poids idéal estimé à partir de la taille, et de hauts niveaux de pression expiratoire positive (PEP, au moins égale à $10 \mathrm{~cm} \mathrm{~d} \mathrm{~d}_{2} \mathrm{O}$ ) doivent être réglés. Les modes ventilatoires en pression ou en volume sont équivalents. Afin de ré-expandre le poumon, des manœuvres de recrutement régulières sont indispensables [6], en étant attentif à leur retentissement hémodynamique. En cas de survenue d'un syndrome de détresse respiratoire aiguë (SDRA), le décubitus ventral est une thérapeutique sûre, qui permet une amélioration de l'oxygénation plus importante que chez le patient non obèse [7]. Enfin, suivant l'extubation, l'application de continuous positive airway pressure (CPAP) ou de VNI serait bénéfique chez le patient obèse morbide.

Par ailleurs, sur le plan cardiovasculaire, les patients obèses morbides présentent, du fait de l'augmentation de la masse graisseuse et du développement d'un syndrome métabolique, une prévalence accrue d'hypertension artérielle et de cardiopathies. Les dysfonctions ventriculaires gauche et droite et une hypertension artérielle pulmonaire pré- et postcapillaire sont fréquentes. De plus, les abords vasculaires peuvent être difficiles, avec une place privilégiée de l'échographie. Les radiographies de thorax au lit, les scanners, l'imagerie par résonance magnétique ou les échographies abdominales sont souvent de réalisation complexe chez les patients obèses morbides. Quant au transport intra- ou extrahospitalier, il nécessite des équipes et du matériel dédiés. De même, les chambres de réanimation doivent être conçues spécifiquement pour accueillir ces patients et l'arrivée d'un 
patient obèse morbide en réanimation doit être anticipée au mieux, avec mise en place d'un mobilier et d'un personnel important pour les soins, formé et entraîné. La toilette est un élément clé et nécessite du temps et de l'organisation, avec une prévention anti-escarres plus fréquente. Le positionnement du patient devra particulièrement prendre soin des zones à risque de compression (matelas à air) avec, si possible, une position demi-assise comme déjà vu plus haut, et des changements de position plus fréquents. Enfin, toute la pharmacocinétique est particulièrement modifiée chez le sujet obèse. Selon le type et la classe de la molécule, les posologies médicamenteuses devront être adaptées au poids idéal, au poids ajusté ou au poids réel.

Ainsi, chez les patients obèses morbides, les durées de ventilation mécanique et de séjour sont augmentées [8], ce qui n'est pas le cas des complications rénales, thrombotiques ou cutanées. Cependant, plusieurs études et méta-analyses montrent que l'obésité pourrait être associée à une diminution de la mortalité, concept appelé «paradoxe de l'obésité » [9]. Ainsi, les patients obèses auraient un meilleur pronostic que les patients non obèses. Cela est plus intuitif qu'il n'y parait lorsqu'on considère que, durant l'évolution humaine, un meilleur statut nutritionnel a permis aux populations de surmonter des périodes de crises. Toutefois, cela n'explique pas le mécanisme ou la nature des facteurs potentiellement protecteurs. Plusieurs hypothèses ont été avancées pour expliquer le bénéfice apparent de l'obésité chez les patients de réanimation, dont l'augmentation des réserves nutritionnelles, les taux plus élevés de cytokines anti-inflammatoires, de cholestérol ou de lipides - qui se lient aux endotoxines et fournissent les précurseurs de synthèse des stéroïdes adrénergiques. De plus, le remodelage diaphragmatique, dû à l'augmentation du poids et de la pression intra-abdominale, est possiblement associé à une diminution de la prévalence du SDRA chez les patients obèses [10].

Cette amélioration du pronostic n'est toutefois pas retrouvée dans certains groupes spécifiques de patients obèses, comme les patients traumatisés ou les patients « super obèses » (IMC $\geq 50 \mathrm{~kg} / \mathrm{m}^{2}$ ). Tout comme pour plusieurs variables biologiques, il y a une relation en U entre la survie et l'IMC, avec cependant une fourchette optimale d'IMC beaucoup plus haute que celle constatée en population générale. Il faut néanmoins garder à l'esprit que l'effet potentiellement bénéfique de l'obésité chez le patient de réanimation n'est peutêtre pas un phénomène réel, mais plutôt le reflet d'un biais de sélection avec des patients atteints de pathologies plus graves (inflammation, séjour prolongé en réanimation, comorbidités préexistantes comme des pathologies malignes) ayant un IMC plus bas et donc un pronostic plus péjoratif [9]. Il y a par conséquent de nombreux facteurs de confusion qui obscurcissent le vrai impact de l'obésité [9].

Si l'effet réel de l'obésité morbide reste encore débattu, il est bien connu depuis plusieurs années qu'un IMC bas est délétère, les patients maigres (IMC $<18,5 \mathrm{~kg} / \mathrm{m}^{2}$ ) ayant le pronostic le plus péjoratif en réanimation. L'anorexie mentale est particulièrement à risque d'augmentation de la mortalité de par ses complications somatiques sévères potentiellement létales et la possibilité de survenue d'un syndrome de renutrition.

Les patients très maigres sont à risque d'hypoglycémies sévères. Un apport intraveineux trop important en glucose chez ces patients ayant un hypo-insulinisme chronique pourra entraîner une sécrétion inappropriée d'insuline responsable d'hypoglycémie réactionnelle. Ces hypoglycémies sont fortement associées à une augmentation de mortalité chez les patients de réanimation. De plus, la perte de poids sévère peut entraîner de nombreuses complications cardiaques : bradycardie sinusale, allongement du QT, insuffisance cardiaque congestive, arythmie. Le risque de décès est principalement lié à la survenue de troubles du rythme cardiaque, particulièrement après administration de catécholamines. La pharmacocinétique est également modifiée pour les IMC très bas et une myopathie sévère est souvent présente.

Le risque majeur spécifique à ces patients reste le syndrome de renutrition, défini par les altérations métaboliques développées suite à une nutrition rapide. La réintroduction de la nutrition à un patient dénutri se traduit par un déclin rapide de la néoglucogenèse et du métabolisme anaérobie. Elle est médiée par l'augmentation rapide de l'insulinémie. L'insuline stimule le mouvement du phosphate, du potassium et du magnésium vers les compartiments intracellulaires. L'hypophosphatémie entraîne un déficit en adénosine triphosphate (ATP) dans toutes les cellules. Les complications potentielles de ces mouvements ioniques et du déficit en ATP incluent une arythmie fatale, une insuffisance cardiaque systolique, une détresse respiratoire, des perturbations hématologiques et des troubles neurologiques.

Ainsi, un monitorage de la renutrition et un régime approprié avec apport de vitamines, minéraux, suivi rapproché et correction des troubles hydro-électrolytiques sont très importants. Une nutrition à faible débit et très hypocalorique (5 kcal/ $\mathrm{kg} /$ jour) est conseillée dans les cas à haut risque de syndrome de renutrition (BMI $<14 \mathrm{~kg} / \mathrm{m}^{2}$, ou privation de nourriture depuis plus de 15 jours), avec un monitorage cardiaque continu.

\section{Conclusion}

Les patients ayant des «poids extrêmes » présentent des spécificités de prise en charge en réanimation, essentiellement respiratoires pour les patients obèses morbides et métaboliques pour les patients maigres. Les idéaux de beauté et la glorification de la minceur stigmatisent les corps « trop gros ». Il est souvent difficile d'accepter que l'obésité n'est 
pas nécessairement mauvaise en réanimation, mais serait en fait plutôt bénéfique, au contraire de la maigreur extrême, qui est franchement délétère. Les causes de cette protection potentiellement induite par le tissu adipeux sont encore mal connues et constituent un champ de recherche considérable.

Liens d'intérêts : S. Jaber est consultant pour Drager, Maquet, Hamilton et Fisher-Paykel France ; A. De Jong n'a pas de lien d'intérêt.

\section{Références}

1. Delay JM, Sebbane M, Jung B, et al (2008) The effectiveness of noninvasive positive pressure ventilation to enhance preoxygenation in morbidly obese patients: a randomized controlled study. Anesth Analg 107:1707-13

2. Futier E, Constantin JM, Pelosi P, et al (2011) Noninvasive ventilation and alveolar recruitment maneuver improve respiratory function during and after intubation of morbidly obese patients: a randomized controlled study. Anesthesiology 114:1354-63

3. De Jong A, Jung B, Jaber S (2014) Intubation in the ICU: we could improve our practice. Crit Care 18:209
4. De Jong A, Molinari N, Conseil M, et al (2014) Video laryngoscopy versus direct laryngoscopy for orotracheal intubation in the intensive care unit: a systematic review and meta-analysis. Intensive care medicine 40:629-39

5. De Jong A, Molinari N, Pouzeratte Y, et al (2015) Difficult intubation in obese patients: incidence, risk factors, and complications in the operating theatre and in intensive care units. $\mathrm{Br} \mathrm{J}$ Anaesth 114:297-306

6. Futier E, Constantin JM, Pelosi P, et al (2010) Intraoperative recruitment maneuver reverses detrimental pneumoperitoneuminduced respiratory effects in healthy weight and obese patients undergoing laparoscopy. Anesthesiology 113:1310-9

7. De Jong A, Molinari N, Sebbane M, et al (2013) Feasibility and effectiveness of prone position in morbidly obese patients with ARDS: a case-control clinical study. Chest 143:1554-61

8. Frat JP, Gissot V, Ragot S, et al (2008) Impact of obesity in mechanically ventilated patients: a prospective study. Intensive care medicine 34:1991-8

9. De Jong A, Jung B, Chanques G, et al (2012) Obesity and mortality in critically ill patients: another case of the simpson paradox? Chest 141:1637-8

10. Gong MN, Bajwa EK, Thompson BT, Christiani DC (2010) Body mass index is associated with the development of acute respiratory distress syndrome. Thorax 65:44-50 